\title{
Formas de fósforo em solos de várzea e biodisponibilidade para o feijoeiro(1)
}

\begin{abstract}
Luiz Arnaldo Fernandes ${ }^{(2)}$, Valdemar Faquin ${ }^{(3)}$, Antonio Eduardo Furtini Neto ${ }^{(3)}$ e Nilton Curi(3)
Resumo - Realizou-se um experimento em casa de vegetação do Departamento de Ciência do Solo da Universidade Federal de Lavras, com o objetivo de verificar a influência da aplicação de calcário e de $\mathrm{P}$ em algumas formas de $\mathrm{P}$ em quatro solos de várzea cultivados com feijoeiro. O delineamento experimental utilizado foi o inteiramente casualizado, em esquema fatorial $4 \times 5 \times 2$, com quatro repetições, a saber: quatro solos [Glei Húmico (GH), Glei Pouco Húmico (GP), Aluvial (A) e Orgânico (O)], cinco doses de $\mathrm{P}\left(75,150,300,500\right.$ e $800 \mathrm{mg} \mathrm{dm}^{-3}$ de P) e dois níveis de calagem (sem e com). Os solos foram incubados em vasos, por 30 dias, com as respectivas doses de calcário, e por mais 150 dias, com as doses de P. No final desse período, foram coletadas amostras de solo para o fracionamento de P, e o restante foi cultivado com feijoeiro. Cada parcela foi constituída por um vaso com $3 \mathrm{dm}^{3}$, em que foram cultivadas duas plantas até o final do ciclo. A calagem e a adubação fosfatada influenciaram as formas de $\mathrm{P}$ de maneira distinta nos solos de várzea estudados; exceto no solo Glei Pouco Húmico, as formas de $\mathrm{P}$ lábil, tanto na ausência quanto na presença de calagem, tiveram pouca participação no $\mathrm{P}$ total dos solos. Na presença da calagem, o P lábil foi a forma de $\mathrm{P}$ preferencialmente absorvida pelas plantas de feijoeiro.
\end{abstract}

Termos para indexação: Phaseolus vulgaris, adubação fosfatada, calagem, fracionamento, nutrição das plantas.

\section{Phosphorus forms in lowland soils and bioavailability to bean plants}

\begin{abstract}
An experiment was carried out in greenhouse of the Soil Science Department of the Universidade Federal de Lavras, State of Minas Gerais, Brazil, with the objective of verifying the influence of application of limestone and phosphorus on some forms of soil $\mathrm{P}$ in four lowland soils cultivated with bean plants. The experimental design utilized was a completely randomized in $4 \times 5 \times 2$ factorial scheme, with four replications, that means: four soils [Gley Humic (GH), Bog Soil (O), Alluvial (A) and Low Humic Gley (GP)], five doses of P (75, 150, 300, 500 and $800 \mathrm{mg} \mathrm{dm}^{-3}$ of P) and two levels of liming (without and with). The soils were incubated during 30 days with limestone and 150 days with phosphorus. After this period, samples of soil were collected to phosphorus fractionation. Each plot was made up of a $3 \mathrm{dm}^{3}$ pot, where two plants were cultivated until the end of the cycle. By the results obtained, it was verified that the application of limestone and $\mathrm{P}$ changed the forms of P in the soils. Except for GP soil, the P labile forms, without and with liming, had a low participation on total phosphorus. Under liming conditions, the P labile is the form of phosphorus preferentially taken up by bean plants.
\end{abstract}

Index terms: Phaseolus vulgaris, phosphate fertilizers, liming, fractionation, plant nutrition.

(1) Aceito para publicação em 21 de agosto de 2001. Extraído da Tese de Doutorado do primeiro autor apresentada à Universidade Federal de Lavras (Ufla). Trabalho financiado pelo CNPq e pela Fapemig.

(2) Universidade Federal de Minas Gerais, Núcleo de Ciências Agrárias, Caixa Postal 135, CEP 39404-006 Montes Claros, MG. E-mail: lafernand@ig.com.br

(3) Ufla, Dep. de Ciência do Solo, Caixa Postal 37, CEP 37200-000 Lavras, MG. Bolsista do CNPq. E-mail: vafaquin@ufla.br, afurtini@ufla.br,niltcuri@ufla.br

\section{Introdução}

O cultivo do feijoeiro em solos de várzea apresenta-se como uma alternativa para maximizar a exploração desses solos, na entressafra do arroz cultivado em solo sob inundação. Andrade (1997) verificou que a baixa disponibilidade de P é o principal fator limitante ao cultivo do feijoeiro, em quatro clas- 
ses de solo de várzea representativas do sul de Minas Gerais.

Quando um solo sob inundação é drenado, ocorre a reoxidação dos constituintes reduzidos do solo com mudanças no $\mathrm{Eh}, \mathrm{pH}$ e concentração de $\mathrm{Fe}^{2+}$, diminuindo a solubilidade tanto do $P$ nativo do solo quanto do P aplicado (Sayal \& De Datta, 1991). Segundo Willett (1979), o decréscimo da disponibilidade de P no solo, com a drenagem de solos inundados, está associada ao aumento na adsorção de $\mathrm{P}$, ocasionado pelo aumento da reatividade dos óxidos de ferro em adsorver P. Desse modo, deficiência de P tem sido verificada em culturas de sequeiro, em rotação com o arroz em solo sob inundação (Willett, 1979; Sah \& Mikkelsen, 1986a, 1986b).

Além das formas inorgânicas de $\mathrm{P}$, as formas orgânicas podem sofrer transformações com os ciclos de umedecimento e secagem do solo. O P orgânico pode se constituir em uma importante fonte desse nutriente às plantas através de sua mineralização. Essa mineralização é mediada pelas fosfatases, enzimas que catalisam a hidrólise de ésteres de fosfatos, liberando fosfato solúvel. Essas enzimas são produzidas pelas plantas e microrganismos do solo, e suas atividades podem ser influenciadas pelos diferentes atributos dos solos (Nahas et al., 1994).

Este trabalho teve o objetivo de verificar a influência da aplicação de calcário e de P sobre algumas formas de $\mathrm{P}$, em quatro solos de várzea cultivados com feijoeiro.

\section{Material e Métodos}

$\mathrm{O}$ experimento foi realizado em condições de casa de vegetação, no Departamento de Ciência do Solo da Universidade Federal de Lavras, em vasos de plástico com capacidade para $3 \mathrm{dm}^{3}$. Cultivou-se o feijoeiro (Phaseolus vulgaris $\mathrm{L}$. cv. Carioca-MG) no período de agosto a novembro de 1997, em amostras de quatro solos de várzea não cultivados anteriormente: Glei Húmico (GH), Orgânico (O), Aluvial (A) e Glei Pouco Húmico (GP); o solo Orgânico foi drenado artificialmente.

As amostras dos solos, coletadas na camada superficial $(0-20 \mathrm{~cm})$ de uma várzea no Município de Lavras, MG, foram destorroadas, secadas ao ar, e passadas em peneira de $5 \mathrm{~mm}$ de abertura. Foram tomadas subamostras e passadas em peneira de $2 \mathrm{~mm}$ de abertura, constituindose, assim, terra fina seca ao ar para caracterizações química, física e mineralógica (Tabela 1).

Pesq. agropec. bras., Brasília, v. 37, n. 3, p. 373-383, mar. 2002
O experimento foi arranjado em esquema fatorial $4 \times 5 \times 2$, com quatro repetições, sendo os fatores: quatro solos (GH, O, A, GP), cinco doses de P $(75,150,300$, $500,800 \mathrm{mg} \mathrm{dm}^{-3}$ ) e dois níveis de calagem (com e sem calcário), em delineamento inteiramente casualizado, com rodízio semanal da posição dos vasos na casa de vegetação. Cada parcela foi constituída por um vaso com $3 \mathrm{dm}^{3}$ de solo.

As doses de $\mathrm{P}$ e calcário foram definidas com base em experimentos anteriores (Andrade, 1997). As doses de calcário para elevar a saturação por bases para, aproximadamente, 50\% corresponderam a 6,4, 4,6, 4,6 e 13,4 tha $\mathrm{t}^{-1}$ para os solos GH, O, A e GP, respectivamente. Utilizou-se um calcário dolomítico calcinado com $35 \%$ de $\mathrm{CaO}, 14 \%$ de $\mathrm{MgO}$ e Poder Relativo de Neutralização Total (PRNT) de $100 \%$.

Os solos foram incubados nos vasos, com e sem as respectivas doses de calcário, por um período de 30 dias, mantendo-se a umidade em torno de $70 \%$ do volume total de poros (VTP) ocupados por água. Após esse período, o material de solo de cada vaso foi secado e peneirado e recebeu a aplicação das respectivas doses de $\mathrm{P}$ e de uma adubação básica de semeadura, com macro e micronutrientes: $70 \mathrm{mg}$ de $\mathrm{N} ; 100 \mathrm{mg}$ de $\mathrm{K} ; 40 \mathrm{mg}$ de S ; 0,5 mg de B; 1,5 mg de Cu e 5,0 mg de $\mathrm{Zn}$ por $\mathrm{dm}^{3}$ de solo. As fontes utilizadas foram reagentes p.a.: $\mathrm{NH}_{4} \mathrm{NO}_{3}$; $\mathrm{NH}_{4} \mathrm{H}_{2} \mathrm{PO}_{4} ; \mathrm{NaH}_{2} \mathrm{PO}_{4} ;\left(\mathrm{NH}_{4}\right)_{2} \mathrm{SO}_{4} ; \mathrm{KNO}_{3} ; \mathrm{H}_{3} \mathrm{BO}_{3}$; $\mathrm{ZnSO}_{4} \cdot 7 \mathrm{H}_{2} \mathrm{O} ; \mathrm{CuCl}_{2} \cdot 5 \mathrm{H}_{2} \mathrm{O}$ e ácido fosfórico $\left(\mathrm{H}_{3} \mathrm{PO}_{3}\right)$. $\mathrm{O}$ material de solo foi novamente incubado por 150 dias, e manteve-se a umidade nos mesmos níveis anteriores.

Após o período de incubação, amostras de solos de cada vaso foram coletadas, para a determinação das formas de P. O fracionamento das formas orgânicas e inorgânicas de P no solo foi realizado conforme o método de Hedley et al. (1982), adaptado por Pavan \& Chaves (1996).

Por esse esquema de fracionamento seqüencial, as seguintes formas de $\mathrm{P}$ lábeis foram caracterizadas: $\mathrm{P}$ extraído pela resina de troca aniônica (P-resina) saturada com bicarbonato; P inorgânico (Pi-bicarb) e orgânico (Pobicarb) adsorvidos nas superfícies dos colóides extraídos com $\mathrm{NaHCO}_{3}$ 0,5 mol L-1 pH 8,5 e P orgânico na biomassa microbiana (Po-microbiano) extraído com $\mathrm{NaHCO}_{3}$ $0,5 \mathrm{~mol} \mathrm{~L}^{-1} \mathrm{pH} \mathrm{8,5}$, após tratamento com clorofórmio. As formas de $\mathrm{P}$ pouco lábil, correspondentes às formas inorgânicas ligadas a Fe e Al (Pi-NaOH), e orgânicas (Po$\mathrm{NaOH}$ ), associadas com compostos húmicos, foram extraídas com $\mathrm{NaOH}$ 0,1 $\mathrm{mol} \mathrm{L}^{-1}$. As formas de $\mathrm{P}$ inorgânicas (Pi-sonif) e orgânicas (Po-sonif), localizadas na superfície interna dos agregados do solo, foram extraídas por ultrasonificação no extrato residual de $\mathrm{NaOH} 0,1 \mathrm{~mol} \mathrm{~L}^{-1}$. 
As formas relativamente insolúveis (P não-lábil), associadas com $\mathrm{Ca}$, foram extraídas com $\mathrm{HCl} 1 \mathrm{~mol} \mathrm{~L}^{-1}$ (Pi-HCl), e o $\mathrm{P}$ residual (P-residual), contendo as formas mais estáveis de $\mathrm{Po}$ e insolúveis de $\mathrm{Pi}$, foi extraído por digestão com $\mathrm{H}_{2} \mathrm{SO}_{4}$ concentrado, e $\mathrm{H}_{2} \mathrm{O}_{2}$. Em todos os extratos de solo, o P foi determinado por colorimetria, conforme Murphy \& Riley (1962).

Cultivaram-se duas plantas de feijoeiro por vaso até a maturação fisiológica dos grãos (estádio R9). Durante o cultivo do feijoeiro, a umidade dos solos foi mantida em torno de $70 \%$ do VTP ocupados por água, por meio de pesagens diárias dos vasos e adição de água deionizada.

As quantidades de $\mathrm{N}$ e $\mathrm{K}$ aplicadas em cobertura foram diferenciadas em razão do crescimento das plantas. Os tratamentos, que proporcionaram, em cada solo, um crescimento normal das plantas, receberam $150 \mathrm{mg}$ de $\mathrm{N}$ e $100 \mathrm{mg}$ de $\mathrm{K}$ por $\mathrm{dm}^{3}$, parcelados em cinco aplicações. Os tratamentos cujas plantas apresentaram menor crescimento receberam coberturas proporcionalmente menores, evitando-se, assim, aplicações excessivas dos nutrientes. No final do ciclo, os grãos foram colhidos separadamente do restante da parte aérea, e foram determinados: a matéria seca dos grãos e a da parte aérea, obtidas em estufa a $70^{\circ} \mathrm{C}$, e a concentração de $\mathrm{P}$ na parte aérea, por digestão nítrico-perclórica (Malavolta et al., 1997).
Todas as variáveis foram submetidas a análise de variância, e equações de regressão foram ajustadas entre essas variáveis (dependentes) e as doses de P aplicadas (variável independente). Substituindo, nessas equações, as doses de $\mathrm{P}$ para $90 \%$ da produção máxima de matéria seca de grãos do feijoeiro (Tabela 2), estimou-se o valor de cada fração de $\mathrm{P}$ correspondente a esse nível de produção, dentro de cada solo e nível de calagem.

\section{Resultados e Discussão}

Nas formas de $\mathrm{P}$ do solo, obtidas pelo fracionamento seqüencial, exceto nas de $\mathrm{P}$ inorgânico (Pisonif) e orgânico (Po-sonif), obtidas por sonificação, P-residual e P não-lábil total, em que ocorreram diferenças significativas $(\mathrm{P}<0,01)$ somente entre os solos estudados, a interação solo $\mathrm{x}$ calagem $\mathrm{x} \mathrm{P}$ foi significativa $(\mathrm{P}<0,01)$.

Pela substituição das doses de $\mathrm{P}$ correspondentes a $90 \%$ da produção máxima de matéria seca de grãos de feijoeiro (MSGR) (Tabela 2 ), nas equações de regressão que relacionaram os teores de $\mathrm{P}$ das formas caracterizadas como variáveis dependentes das doses de P aplicadas, em cada solo e nível de calagem,

Tabela 1. Principais atributos químicos, físicos e mineralógicos dos solos antes da aplicação dos tratamentos ${ }^{(1)}$.

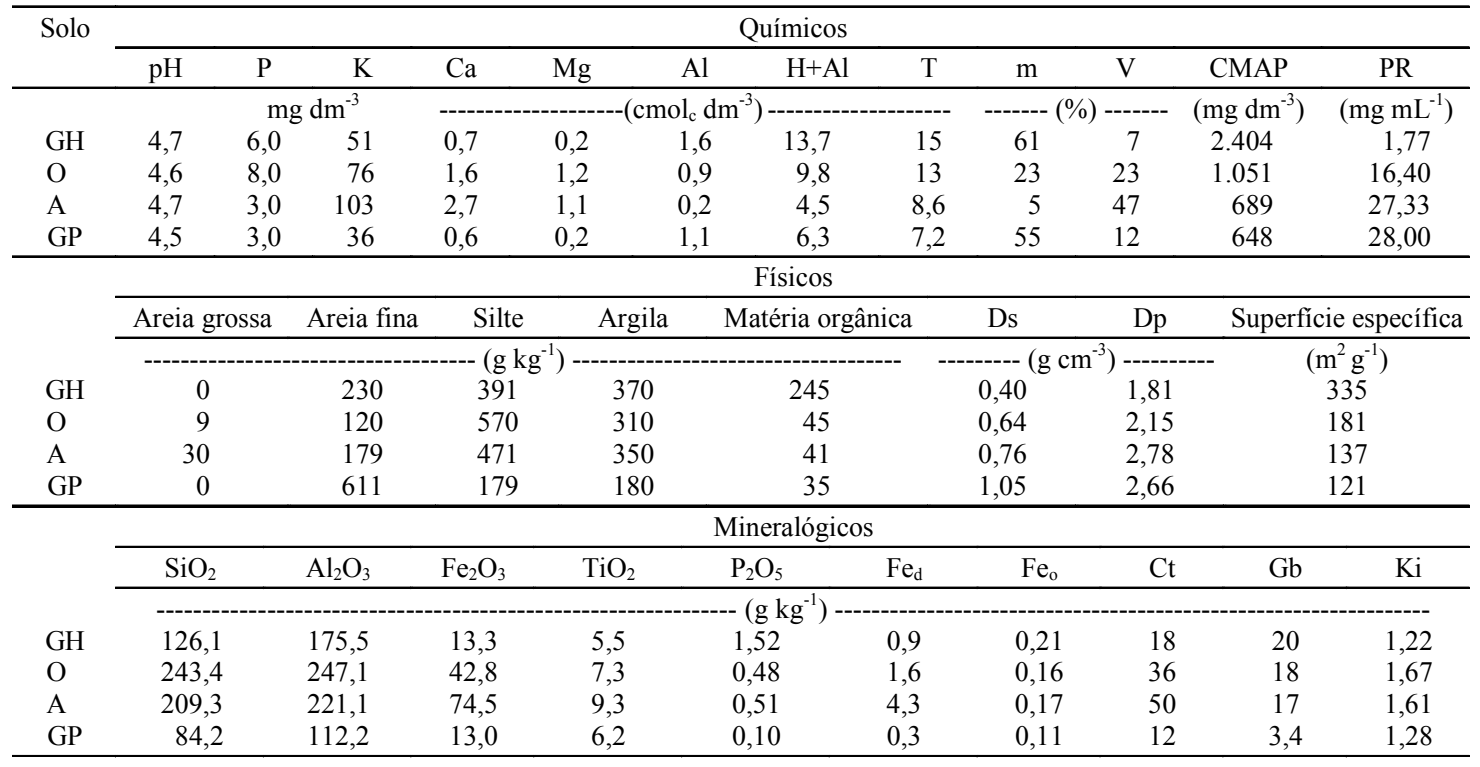

(1)GH: Glei Húmico; O: Orgânico; A: Aluvial; GP: Glei Pouco Húmico; pH: pH em água; P: fósforo pelo Mehlich 1; K: potássio; Ca: cálcio; Mg: magnésio; Al: alumínio; $\mathrm{H}+\mathrm{Al}$ : acidez potencial; T: capacidade de troca de cátions a pH 7,0; m: saturação por Al; V: saturação por bases; CMAP: capacidade máxima de adsorção de fósforo (Alvarez \& Fonseca, 1990); PR: fósforo remanescente; Ds: densidade do solo; Dp: densidade de partículas (Embrapa, 1997); $\mathrm{Fe}_{\mathrm{d}}$ : ferro ditionito; $\mathrm{Fe}_{\mathrm{o}}$ : ferro oxalato; Ct: caulinita; $\mathrm{Gb}$ : gibbsita (Mehra \& Jackson, 1960) determinados na fração argila e corrigidos para terra fina; Ki: relação molecular $\mathrm{SiO}_{2} / \mathrm{Al}_{2} \mathrm{O}_{3}$. 
estimaram-se os valores de $\mathrm{P}$ relativos às diversas formas correspondentes a esse nível de produção. Nas formas Pi-sonif, Po-sonif, P-residual e P não-lábil, considerou-se, para estimar esses valores, a média dos respectivos valores obtidos em cada dose de $\mathrm{P}$ aplicada em cada solo e nível de calagem, uma vez que não houve diferenças significativas entre as doses de $\mathrm{P}$ aplicadas.

$\mathrm{Na}$ ausência de calagem, os valores de $\mathrm{P}$ lábil total (P-resina + Po-microb + Pi-bicarb + Po-bicarb) dos solos decresceram na seguinte ordem: $\mathrm{GP}>\mathrm{GH}>$ $\mathrm{A}>\mathrm{O}$ (Tabela 3). Embora nos solos GH e GP os valores de $\mathrm{P}$ lábil total tenham sido superiores, as produções de matéria seca de grãos (MSGR), na dose de $\mathrm{P}$ correspondente a $90 \%$ da produção máxima, foram significativamente menores em relação às estimadas nos solos $\mathrm{O}$ e A (Tabela 2). Talvez, os maiores teores de $\mathrm{Al}$ trocável e menores teores de $\mathrm{Ca} \mathrm{e}$ Mg dos solos GH e GP (Tabela 1) tenham sido a causa da menor produção de MSGR nesses solos.

Os valores de $\mathrm{P}$ lábil total dos solos decresceram, com a aplicação de calcário, na seguinte ordem: GP > $\mathrm{A}>\mathrm{O}>\mathrm{GH}$ (Tabela 3). Com a calagem, o valor de $\mathrm{P}$ lábil total, na dose de $\mathrm{P}$ correspondente a $90 \%$ da produção máxima, reduziu-se em $25 \%$ e $12 \%$ nos solos GH e GP, respectivamente, enquanto, nesta mesma dose de $\mathrm{P}$, a produção de MSGR aumentou em $395 \%$ e $455 \%$, respectivamente (Tabela 2). Nos solos $\mathrm{O}$ e A, na dose de $\mathrm{P}$ correspondente a $90 \%$ da máxima produção, os valores de $\mathrm{P}$ lábil au- mentaram $7 \%$ e $10 \%$, respectivamente, em relação ao obtido na ausência de calagem, enquanto a produção de MSGR aumentou $43 \%$ e $65 \%$, respectivamente, o que evidencia o efeito tóxico do $\mathrm{Al}$ e/ou os baixos teores de $\mathrm{Ca}$ e $\mathrm{Mg}$ no solo em que se produziu o feijoeiro.

Em todos os solos, independentemente da calagem, houve aumento do $\mathrm{P}$ lábil total com o incremento das doses de $\mathrm{P}$, e as formas de $\mathrm{P}$ inorgânico (P-resina e Pi-bicarb) foram as que mais contribuíram para o P lábil total. Pavan \& Chaves (1996), em Latossolo, e Ivanoff et al. (1998), em Histosols, também verificaram uma pequena contribuição do Po-microb do $\mathrm{P}$ lábil total. Segundo Hedley et al. (1982), as formas de P inorgânico lábil são as que mais contribuem para o $\mathrm{P}$ lábil total em solos que receberam adubações com fertilizantes minerais. Também Beck (1991), estudando as transformações do $P$ inorgânico e P orgânico após 18 anos de cultivo de um Ultisol da região amazônica, verificou aumento significativo do $\mathrm{P}$ lábil nas parcelas que receberam aplicação de fertilizantes fosfatados.

$\mathrm{Na}$ ausência de calagem, os valores de Pi-bicarb, Po-bicarb e $\mathrm{P}$ lábil total correlacionaram-se significativa e negativamente com as produções de MSGR, matéria seca total da parte aérea (MSTO) e P acumulado na MSTO (P-MSTO), correspondentes à dose de P com $90 \%$ da produção máxima (Tabela 4). Esse comportamento pode estar relacionado aos elevados teores de $\mathrm{Al}$ trocável e baixos teores de $\mathrm{Ca}$ e $\mathrm{Mg}$,

Tabela 2. Equações de regressão ajustadas entre a produção de grãos como variável dependente (Y) das doses de fósforo aplicadas (X), produção de grãos correspondentes a 90\% da produção máxima e doses de P correspondente a 90\% da produção máxima.

\begin{tabular}{|c|c|c|c|c|}
\hline Solo $^{(1)}$ & Equação & $\mathrm{R}^{2}$ & $\begin{array}{c}90 \% \text { produção máxima } \\
\text { de grãos } \\
\left(\mathrm{g} \mathrm{vaso}^{-1}\right)\end{array}$ & $\begin{array}{c}\text { Dose de P para } 90 \% \text { da } \\
\text { produção máxima } \\
\left(\mathrm{mg} \mathrm{dm}^{-3}\right)\end{array}$ \\
\hline \multicolumn{5}{|c|}{ Sem calagem } \\
\hline GH & $Y=-0,8805+0,0061 * * X$ & 0,96 & 3,62 & 735 \\
\hline $\mathrm{O}$ & $\mathrm{Y}=-3,01+1,5102 * * \mathrm{X}^{0,5}-0,0294 * * \mathrm{X}$ & 0,98 & 14,77 & 333 \\
\hline A & $Y=-5,63+1,6838 * * X^{0,5}-0,0343 * * X$ & 0,99 & 13,55 & 322 \\
\hline GP & $\mathrm{Y}=-4,99+0,7547 * * \mathrm{X}^{0,5}-0,0145 * * \mathrm{X}$ & 0,99 & 4,37 & 412 \\
\hline \multicolumn{5}{|c|}{ Com calagem } \\
\hline $\mathrm{GH}$ & $Y=-21,12+2,9811 * * X^{0,5}-0,0541 * * X$ & 0,99 & 17,89 & 460 \\
\hline $\mathrm{O}$ & $Y=1,730+1,6894 * * X^{0,5}-0,0328 * * X$ & 0,98 & 21,14 & 299 \\
\hline A & $Y=-12,85+3,0192 * * X^{0,5}-0,0604 * * X$ & 0,99 & 22,38 & 345 \\
\hline GP & $Y=-9,03+2,7582 * * X^{0,5}-0,0529 * * X$ & 0,99 & 24,23 & 359 \\
\hline
\end{tabular}

${ }^{(1)} \mathrm{GH}$ : Glei Húmico; O: Orgânico; A: Aluvial; GP: Glei Pouco Húmico. **Significatico a 1\% de probabilidade pelo teste t. 
Tabela 3. Equações de regressão ajustadas entre o $\mathrm{P}$ lábil (Y) em diversas formas, $\mathrm{mg} \mathrm{dm}^{-3}$, como variável dependente das doses de $\mathrm{P}$ aplicadas $(\mathrm{X}), \mathrm{mg} \mathrm{dm}^{-3}$, e valores de $\mathrm{P}$ lábil correspondentes a $90 \%$ da produção máxima de matéria seca de grãos (P lábil - 90\%).

\begin{tabular}{|c|c|c|c|c|}
\hline Solo $^{(1)}$ & Extrator & Equação & $\mathrm{R}^{2}$ & P lábil - 90\% \\
\hline & & & gem & \\
\hline \multirow[t]{12}{*}{ GH } & P-resina & $\mathrm{Y}=0,6182+0,0596 * * \mathrm{X}$ & 0,98 & 43,21 \\
\hline & Po-microbiano & $\mathrm{Y}=2,3087+0,0047 * * \mathrm{X}$ & 0,88 & 5,76 \\
\hline & Pi-bicarb & $\mathrm{Y}=-0,0398+0,0549 * * \mathrm{X}$ & 0,98 & 40,31 \\
\hline & Po-bicarb & $\mathrm{Y}=2,7198+0,0009 * * \mathrm{X}$ & 0,89 & 3,38 \\
\hline & $\mathrm{P}$ lábil total & $\mathrm{Y}=4,3706+0,1202 * * \mathrm{X}$ & 0,99 & 92,70 \\
\hline & & \multicolumn{3}{|c|}{ Com calagem } \\
\hline & P-resina & $\mathrm{Y}=7,4326+0,060 * * \mathrm{X}$ & 0,94 & 35,05 \\
\hline & Po-microbiano & $\mathrm{Y}=-2,3007+0,0031 * * \mathrm{X}$ & 0,90 & 3,71 \\
\hline & Pi-bicarb & $Y=-0,9098+0,0636^{* *} \mathrm{X}$ & 0,99 & 28,36 \\
\hline & Po-bicarb & $\mathrm{Y}=2,6774+0,0006^{* * \mathrm{X}}$ & 0,96 & 2,97 \\
\hline & $\mathrm{P}$ lábil total & $\mathrm{Y}=12,035+0,1258 * * \mathrm{X}$ & 0,99 & 69,96 \\
\hline & & & gem & \\
\hline \multirow[t]{11}{*}{$\mathrm{O}$} & P-resina & $\mathrm{Y}=1,2284+0,1169 * \mathrm{X}$ & 0,99 & 40,16 \\
\hline & Po-microbiano & $\mathrm{Y}=21274+0,0011 * * \mathrm{X}$ & 0,91 & 2,49 \\
\hline & Pi-bicarb & $\mathrm{Y}=-3,9484+0,0798 * * \mathrm{X}$ & 0,99 & 22,36 \\
\hline & Po-bicarb & $\mathrm{Y}=1,1471+0,0036^{* * \mathrm{X}}$ & 0,99 & 2,35 \\
\hline & P lábil total & $\mathrm{Y}=2,2582+0,1955^{* *} \mathrm{X}$ & 0,99 & 67,36 \\
\hline & & \multicolumn{3}{|c|}{ Com calagem } \\
\hline & P-resina & $\mathrm{Y}=-1,4750+0,1482 * * \mathrm{X}$ & 0,99 & 42,84 \\
\hline & Po-microbiano & $\mathrm{Y}=2,2397+0,0097 * * \mathrm{X}$ & 0,99 & 5,14 \\
\hline & Pi-bicarb & $\mathrm{Y}=-1,8190+0,0761 * * \mathrm{X}$ & 0,99 & 20,93 \\
\hline & Po-bicarb & $\mathrm{Y}=2,9013+0,0008 * * \mathrm{X}$ & 0,95 & 3,14 \\
\hline & $\mathrm{P}$ lábil total & $\mathrm{Y}=2,2984+0,2333 * * \mathrm{X}$ & 0,99 & 72,05 \\
\hline \multirow[t]{12}{*}{ A } & & \multicolumn{3}{|c|}{ Sem calagem } \\
\hline & P-resina & $\mathrm{Y}=0,8319+0,1473 * * \mathrm{X}$ & 0,99 & 48,26 \\
\hline & Po-microbiano & $\mathrm{Y}=2,2937+0,0079 * * \mathrm{X}$ & 0,99 & 4,84 \\
\hline & Pi-bicarb & $\mathrm{Y}=1,8158+0,0563^{*} * \mathrm{X}$ & 0,99 & 19,94 \\
\hline & Po-bicarb & $\mathrm{Y}=2,3582+0,0026 * * \mathrm{X}$ & 0,97 & 3,20 \\
\hline & $\mathrm{P}$ lábil total & $\mathrm{Y}=7,2975+0,1998 * * \mathrm{X}$ & 0,99 & 76,24 \\
\hline & & \multicolumn{3}{|c|}{ Com calagem } \\
\hline & P-resina & $\mathrm{Y}=7,3782+0,1257 * * \mathrm{X}$ & 0,99 & 50,74 \\
\hline & Po-microbiano & $\mathrm{Y}=2,8500+0,0072 * * \mathrm{X}$ & 0,99 & 5,37 \\
\hline & Pi-bicarb & $\mathrm{Y}=2,5988+0,0619^{*} * \mathrm{X}$ & 0,98 & 23,95 \\
\hline & Po-bicarb & $\mathrm{Y}=2,2804+0,0043 * * \mathrm{X}$ & 0,99 & 3,76 \\
\hline & $\mathrm{P}$ lábil total & $\mathrm{Y}=15,1073+0,1991^{* *} \mathrm{X}$ & 0,99 & 83,82 \\
\hline \multirow[t]{12}{*}{ GP } & & \multicolumn{3}{|c|}{ Sem calagem } \\
\hline & P-resina & $\mathrm{Y}=0,9689+0,1520 * * \mathrm{X}$ & 0,99 & 63,59 \\
\hline & Po-microbiano & $\mathrm{Y}=2,1361+0,0046^{* *} \mathrm{X}$ & 0,89 & 4,03 \\
\hline & Pi-bicarb & $\mathrm{Y}=0,0005+0,0808^{*} * \mathrm{X}$ & 0,99 & 33,29 \\
\hline & Po-bicarb & $\mathrm{Y}=0,0415+0,0049 * * \mathrm{X}$ & 0,99 & 2,06 \\
\hline & $\mathrm{P}$ lábil total & $\mathrm{Y}=3,1471+0,2424 * * \mathrm{X}$ & 0,99 & 107,97 \\
\hline & & \multicolumn{3}{|c|}{ Com calagem } \\
\hline & P-resina & $\mathrm{Y}=3,3255+0,1560 * * \mathrm{X}$ & 0,99 & 59,33 \\
\hline & Po-microbiano & $\mathrm{Y}=2,3293+0,0046 * * \mathrm{X}$ & 0,95 & 3,98 \\
\hline & Pi-bicarb & $\mathrm{Y}=-0,9079+0,0846^{* *} \mathrm{X}$ & 0,99 & 29,46 \\
\hline & Po-bicarb & $\mathrm{Y}=0,3016+0,0057 * * \mathrm{X}$ & 0,99 & 2,35 \\
\hline & $\mathrm{P}$ lábil total & $\mathrm{Y}=5,0486+0,2510 * * \mathrm{X}$ & 0,99 & 95,12 \\
\hline
\end{tabular}

${ }^{(1)} \mathrm{GH}$ : Glei Húmico; O: Orgânico; A: Aluvial; GP: Glei Pouco Húmico. **Significativo a 1\% de probabilidade pelo teste de t. 
que, provavelmente, limitaram a produção do feijoeiro. Por outro lado, quando o Al trocável foi neutralizado pela calagem, as produções de MSGR, MSTO e P-MSTO correlacionaram-se significativa e positivamente com os valores de P-resina, Po-bicarb e $\mathrm{P}$ lábil total, correspondentes à dose de $\mathrm{P}$ com $90 \%$ da produção máxima.

O P-resina (Amer et al., 1955; Sibbesen, 1977) e o Pi-bicarb (Browman \& Cole, 1978; Tiessen et al., 1992) são considerados as formas mais disponíveis de $\mathrm{P}$ às plantas. O Po-bicarb, segundo Stewart \& Tiessen (1987), tem importante papel na ciclagem do P, através dos processos de mineralização e imobilização, pois é uma fonte de $\mathrm{P}$ disponível a curto prazo para as plantas.

$\mathrm{Na}$ ausência de calagem, os valores de $\mathrm{P}$ pouco lábil total nos solos decresceram na seguinte ordem: $\mathrm{GH}>\mathrm{A}>\mathrm{O}>\mathrm{GP}$ (Tabela 5). Por sua vez, com a aplicação de calcário, a seqüência dos solos foi a seguinte: $\mathrm{GH}>\mathrm{O}>\mathrm{A}>\mathrm{GP}$. Verificou-se, também, que os valores de $\mathrm{P}$ pouco lábil total aumentaram com a aplicação de calcário nos solos $\mathrm{GH}$ e $\mathrm{O}$, enquanto nos solos A e GP ocorreu uma redução.
Os valores de $\mathrm{P}$ pouco lábil total correspondentes à dose de $\mathrm{P}$ com $90 \%$ da produção máxima, nos solos $\mathrm{GH}$ e $\mathrm{O}$ com calagem, foram $34 \%$ e $37 \%$, respectivamente, superiores aos estimados na ausência de calagem. Diferentemente, nos solos A e GP com calagem foram $22 \%$ e $44 \%$, respectivamente, inferiores aos estimados na ausência de calagem, comportamento esperado em solos ácidos que receberam calagem.

$\mathrm{O}$ aumento nos teores de $\mathrm{Pi}-\mathrm{NaOH}$ e $\mathrm{Po}-\mathrm{NaOH}$ causou o aumento do $\mathrm{P}$ pouco lábil, uma vez que, tanto na ausência quanto na presença da calagem, foram as formas que mais contribuíram para o $\mathrm{P}$ pouco lábil total. $\mathrm{O}$ hidróxido de sódio extrai formas de $P$ consideradas de baixa disponibilidade para as plantas associadas a fosfatos amorfos e cristalinos de $\mathrm{Fe}$ e Al (Willians et al., 1980). Neste sentido, Wang et al. (1977) verificaram que a forma $\mathrm{Pi}-\mathrm{NaOH}$ foi a que mais se alterou com o cultivo da cana-de-açúcar. Segundo Tiessen et al. (1992), em solos tropicais o $\mathrm{Pi}-\mathrm{NaOH}$ é uma forma relativamente dinâmica. No presente estudo, na presença de calagem foi

Tabela 4. Coeficientes de correlação entre os valores de $P$ nas diversas formas com a produção de matéria seca de grãos (MSGR), matéria seca total da parte aérea (MSTO) e fósforo acumulado na MSTO (P-MSTO), nas doses de P correspondentes a $90 \%$ da produção máxima de matéria seca de grãos, nos solos estudados, na ausência e presença de calagem

\begin{tabular}{|c|c|c|c|c|c|c|c|}
\hline Variável & P-resina & P-microb & Pi-bicarb & Po-bicarb & $\mathrm{P}$ lábil & $\mathrm{Pi}-\mathrm{NaOH}$ & Po-NaOH \\
\hline & & & & em calagem & & & \\
\hline MSGR & $-0,49^{\mathrm{ns}}$ & $-0,19^{\mathrm{ns}}$ & $-0,95 * *$ & $-0,80^{*}$ & $-0,91 * *$ & $-0,49^{\mathrm{ns}}$ & $-0,11^{\mathrm{ns}}$ \\
\hline MSTO & $-0,54^{\mathrm{ns}}$ & $-0,13^{\mathrm{ns}}$ & $-0,93 * *$ & $-0,83^{*}$ & $-0,93 * *$ & $-0,43^{\mathrm{ns}}$ & $-0,04^{\mathrm{ns}}$ \\
\hline \multirow[t]{2}{*}{ P-MSTO } & $-0,49^{\mathrm{ns}}$ & $-0,01^{\mathrm{ns}}$ & $-0,94 * *$ & $-0,72 *$ & $-0,89 * *$ & $-0,40^{\mathrm{ns}}$ & $-0,04^{\mathrm{ns}}$ \\
\hline & & & & Com calagem & & & \\
\hline MSGR & $0,98 * *$ & $-0,23^{\mathrm{ns}}$ & $0,03^{\mathrm{ns}}$ & $0,92 * *$ & $0,90 * *$ & $-0,97 * *$ & $-0,98 * *$ \\
\hline MSTO & $0,95^{* *}$ & $-0,64^{\mathrm{ns}}$ & $0,49^{\mathrm{ns}}$ & $0,92 * *$ & $0,98 * *$ & $-0,74^{*}$ & $-0,82 *$ \\
\hline \multirow[t]{3}{*}{ P-MSTO } & $0,99 * *$ & $-0,40^{\mathrm{ns}}$ & $0,24^{\mathrm{ns}}$ & $0,97 * *$ & $0,97 * *$ & $-0,89 * *$ & $-0,95 * *$ \\
\hline & Pi-sonif & Po-sonif & P p. lábil & $\mathrm{Pi}-\mathrm{HCl}$ & P-residual & P não-lábil & P total \\
\hline & & & & Sem calagem & & & \\
\hline MSGR & $-0,04^{\mathrm{ns}}$ & $-0,61^{\mathrm{ns}}$ & $-0,46^{\mathrm{ns}}$ & $-0,54^{\mathrm{ns}}$ & $-0,37^{\mathrm{ns}}$ & $-0,37^{\mathrm{ns}}$ & $-0,43^{\mathrm{ns}}$ \\
\hline MSTO & $0,02^{\mathrm{ns}}$ & $-0,55^{\mathrm{ns}}$ & $-0,40^{\mathrm{ns}}$ & $-0,55^{\mathrm{ns}}$ & $-0,31^{\mathrm{ns}}$ & $-0,31^{\mathrm{ns}}$ & $-0,37^{\mathrm{ns}}$ \\
\hline \multirow[t]{2}{*}{ P-MSTO } & $0,13^{\mathrm{ns}}$ & $-0,54^{\mathrm{ns}}$ & $-0,36^{\mathrm{ns}}$ & $-0,48^{\mathrm{ns}}$ & $-0,28^{\mathrm{ns}}$ & $-0,28^{\mathrm{ns}}$ & $-0,34^{\mathrm{ns}}$ \\
\hline & & & & Com calagem & & & \\
\hline MSGR & $-0,90 * *$ & $-0,89 * *$ & $-0,98 * *$ & $-0,92 * *$ & $-0,97 * *$ & $-0,97 * *$ & $-0,97 * *$ \\
\hline MSTO & $-0,79^{*}$ & $-0,57^{\mathrm{ns}}$ & $-0,76^{*}$ & $-0,67^{\mathrm{ns}}$ & $-0,76^{*}$ & $-0,76^{*}$ & $-0,75^{*}$ \\
\hline P-MSTO & $-0,85^{*}$ & $-0,77^{*}$ & $-0,91 * *$ & $-0,82 *$ & $-0,90 * *$ & $-0,89 * *$ & $-0,90 * *$ \\
\hline
\end{tabular}

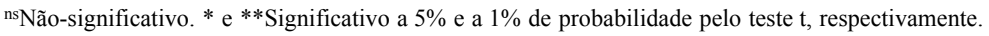


Tabela 5. Equações de regressão ajustadas entre o $\mathrm{P}$ pouco lábil $(\mathrm{Y})$ em diversas formas, $\mathrm{mg} \mathrm{dm}^{-3}$, como variável dependente das doses de $\mathrm{P}$ aplicadas $(\mathrm{X}), \mathrm{mg} \mathrm{dm}^{-3}$, e valores de $\mathrm{P}$ pouco lábil correspondentes a $90 \%$ da produção máxima de matéria seca de grãos (P pouco lábil - 90\%).

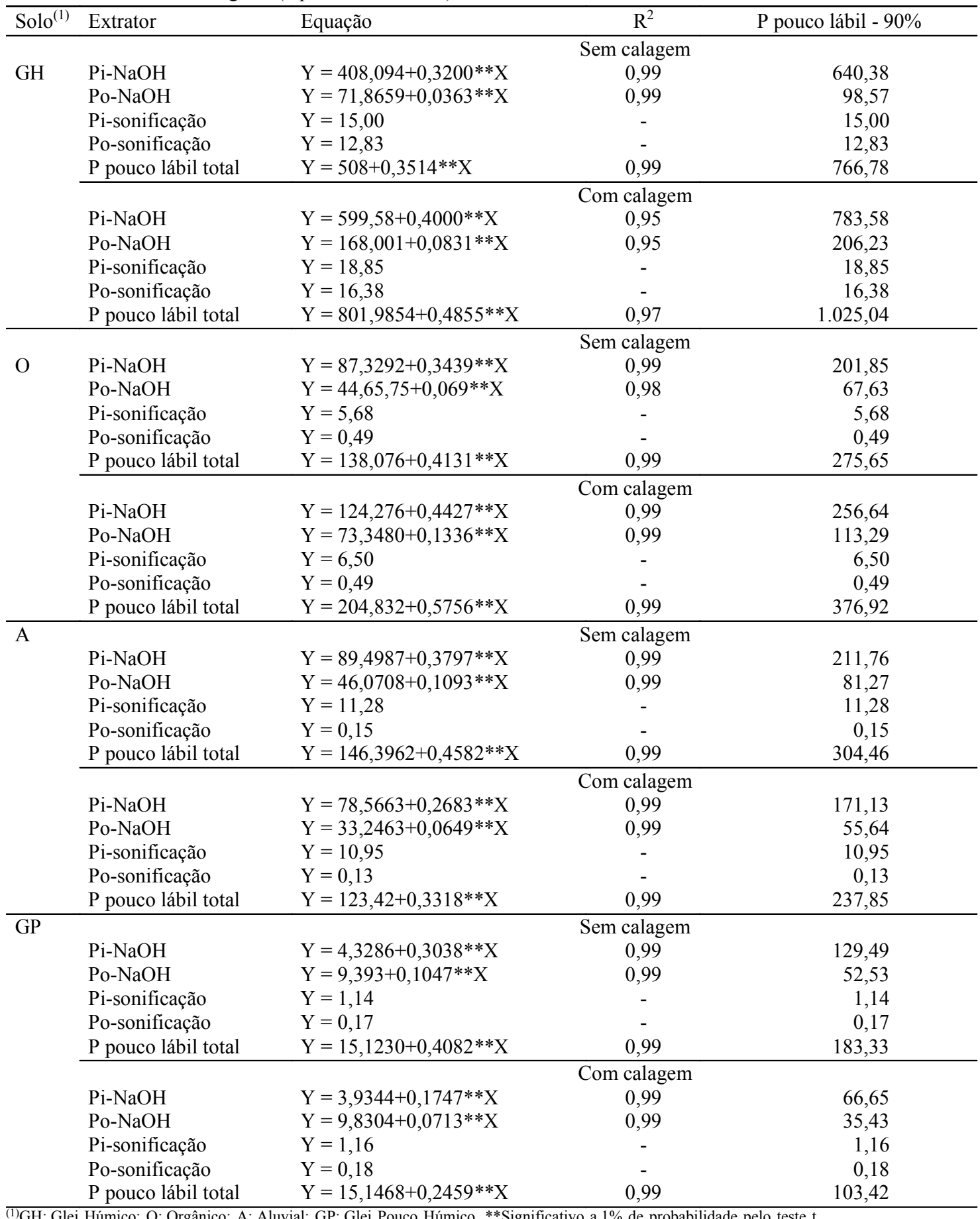


verificada correlação significativa e negativa entre as produções de MSGR, MSTO e P-MSTO com os valores de $\mathrm{P}$ pouco lábil das diversas formas, correspondentes a $90 \%$ da produção máxima (Tabela 4), o que indica que essas formas não contribuíram para a nutrição das plantas de feijoeiro. De acordo com Lindsay \& Moreno (1960), a solubilidade de compostos fosfatados depende do $\mathrm{pH}$ do solo. Em $\mathrm{pH}$ acima de 7,0, a solubilidade é controlada por fosfatos de cálcio, e em $\mathrm{pH}$ abaixo desse valor, por fosfatos de ferro e alumínio. Segundo Swenson et al. (1949), para que uma quantidade significativa de $P$ seja liberada dos compostos de $\mathrm{Fe}$ e $\mathrm{Al}$, o $\mathrm{pH}$ do solo deve ser elevado para valores acima de 7,0.

$\mathrm{O}$ aumento das formas $\mathrm{Pi}-\mathrm{NaOH}$ e $\mathrm{Po}-\mathrm{NaOH}$ nos solos GH e O com aplicação de calcário se deve, provavelmente, à oxidação da matéria orgânica e ao aumento dos teores de $\mathrm{Al}$ amorfo nesses solos durante o período de incubação, uma vez que, segundo Farmer \& Mitchell (1963), durante a oxidação da matéria orgânica do solo pode ocorrer a formação de Fe e Al amorfo.

Fernandes et al. (1998), utilizando o fracionamento de Chang \& Jackson (1957), também verificaram um aumento nos teores de P extraído pelo $\mathrm{NaOH}$ quando se aplicou calcário. Segundo os autores, com a aplicação de calcário ocorre uma dispersão dos vários compostos presentes no solo pela elevação do $\mathrm{pH}$, expondo cargas elétricas positivas que adsorvem $\mathrm{P}$, e que podem ser extraídos pelos métodos utilizados na determinação dessas formas de P. Além disso, com a calagem pode haver formação de precipitados de $\mathrm{Al}$ que podem adsorver $\mathrm{P}$, como discutido anteriormente.

Em todos os solos, tanto na ausência quanto na presença de calagem, houve aumento das formas de $\mathrm{Pi}-\mathrm{NaOH}$ e Po-NaOH com o incremento das doses de P (Tabela 5). Araújo et al. (1993) também verificaram variações nas formas de $\mathrm{P}$ extraídas pelo hidróxido de sódio em solos fertilizados com $\mathrm{P}$ e cultivados com cana-de-açúcar, já que a forma de $\mathrm{Po}-\mathrm{NaOH}$ é maior na área de cultivo do que na área sob vegetação nativa. Nas formas Pi-sonif e Po-sonif, ocorreram diferenças significativas somente entre os solos estudados (Tabela 5).

Os valores de $\mathrm{P}$ não-lábil ( $\mathrm{Pi}-\mathrm{HCl}+\mathrm{P}$-residual) nos solos decresceram na seguinte ordem:
$\mathrm{GH}>\mathrm{O} \cong \mathrm{A}>\mathrm{GP}$ (Tabela 6). Para essa forma de $\mathrm{P}$, ocorreram diferenças significativas apenas entre os solos estudados, e a forma P-residual, nos quatro solos estudados foi a que mais contribuiu para o $\mathrm{P}$ nãolábil total. Segundo Araújo et al. (1993) e Pavan \& Chaves (1996), este comportamento é esperado em solos ácidos.

$\mathrm{A}$ forma $\mathrm{Pi}-\mathrm{HCl}$ corresponde às formas de $\mathrm{P}$ ligadas a Ca (Walker \& Syers, 1976), e em solos não fertilizados de regiões temperadas está associada às apatitas, e é considerada disponível a longo prazo mediante o intemperismo do solo (Smeck, 1985). Segundo Araújo et al. (1993), em solos ácidos dos trópicos esta forma de $\mathrm{P}$ ligada a Ca não é tão estável, e é disponível, a médio prazo, às plantas. Estes autores verificaram, também, que a forma P-residual apresentou pequenas variações após 25 anos de cultivo do solo com cana-de-açúcar, indicando, assim, que muito pouco do fertilizante acumulou-se nessa forma de fósforo. A forma P-residual representa o $\mathrm{P}$ ocluso, retido pela hematita, goetita e gibbsita (Smeck, 1985).

$\mathrm{Na}$ ausência de calagem os valores de $\mathrm{P}$ total ( $\mathrm{P}$ lábil $+\mathrm{P}$ pouco lábil $+\mathrm{P}$ não-lábil) nos solos decresceram na seguinte ordem: $\mathrm{GH}>\mathrm{A}>\mathrm{O}>\mathrm{GP}$ (Tabela 6). No entanto, na presença de calcário, a ordem foi a seguinte: $\mathrm{GH}>\mathrm{O}>\mathrm{A}>\mathrm{GP}$. Os maiores valores de $\mathrm{P}$ total, na dose correspondente a $90 \%$ da produção máxima no solo $\mathrm{O}$ em relação ao solo $\mathrm{A}$, na presença de calagem, se devem a um aumento do $\mathrm{P}$ pouco lábil com a aplicação de calcário nesse solo, e a uma redução dessa forma no solo A.

Tanto na presença quanto na ausência de calagem, em todos os solos estudados, na dose correspondente a $90 \%$ da máxima produção, ocorreu um aumento nos valores de P total com o incremento das doses de P (Tabela 6). Segundo Perrott \& Mansell (1989), a aplicação de calcário e de $\mathrm{P}$ provocam um rearranjamento das formas de $\mathrm{P}$ dos solos.

Na dose de $\mathrm{P}$ correspondente a $90 \%$ da produção máxima, o P lábil total (Tabela 3) correspondeu a 5,2\%, $11,2 \%, 11,7 \%$ e $32,8 \%$ do P total (Tabela 6), respectivamente, nos solos GH, O, A e GP, sem calagem. $\mathrm{Na}$ presença de calagem, esta forma de $\mathrm{P}$ correspondeu a $3,5 \%, 10,2 \%, 14,4 \%$ e $40,1 \%$ do $\mathrm{P}$ total, respectivamente, em GH, O, A e GP. Verifica-se, 
exceto no solo GP, uma pequena participação do $\mathrm{P}$ lábil total no $\mathrm{P}$ total. Ivanoff et al. (1998) também verificaram uma pequena participação do $\mathrm{P}$ lábil no $\mathrm{P}$ total em Histosols de Everglades, Sul da Florida, EUA.
Os valores de $\mathrm{P}$ pouco lábil total (soma das frações de $\mathrm{P}$ pouco lábil na dose correspondente a $90 \%$ da produção máxima) (Tabela 5), correspondentes à dose de $\mathrm{P}$ com $90 \%$ da produção máxima, nos quatro

Tabela 6. Equações de regressão ajustadas entre o P não-lábil (Y) em diversas formas, $\mathrm{mg} \mathrm{dm}^{-3}$, como variável dependente das doses de $\mathrm{P}$ aplicadas $(\mathrm{X}), \mathrm{mg} \mathrm{dm}^{-3}$, e valores de $\mathrm{P}$ não-lábil correspondentes a $90 \%$ da produção máxima de matéria seca de grãos (P não-lábil - 90\%).

\begin{tabular}{|c|c|c|c|c|}
\hline $\mathrm{Solo}^{(1)}$ & Extrator & Equação & $\mathrm{R}^{2}$ & P não-lábil - 90\% \\
\hline & & & & \\
\hline \multirow[t]{10}{*}{$\mathrm{GH}$} & $\mathrm{Pi}-\mathrm{HCl}$ & $\mathrm{Y}=6,3387+0,0044 * * \mathrm{X}$ & 0,99 & 9,57 \\
\hline & P-residual & $\mathrm{Y}=898,06$ & - & 898,06 \\
\hline & $\mathrm{P}$ não-lábil total & $Y=907,63$ & - & 907,63 \\
\hline & $P$ total & $\mathrm{Y}=1421,87+0,4700 * * \mathrm{X}$ & 0,98 & $1.767,11$ \\
\hline & & \multicolumn{3}{|c|}{ Com calagem } \\
\hline & $\mathrm{Pi}-\mathrm{HCl}$ & $\mathrm{Y}=7,5997+0,0031 * * \mathrm{X}$ & 0,98 & 9,03 \\
\hline & P-residual & $\mathrm{Y}=893,01$ & - & 893,01 \\
\hline & $\mathrm{P}$ não-lábil total & $\mathrm{Y}=902,04$ & - & 902,04 \\
\hline & P total & $\mathrm{Y}=1741,17+0,5625 * * \mathrm{X}$ & 0,98 & $2.000,00$ \\
\hline & & & & \\
\hline \multirow[t]{9}{*}{$\mathrm{O}$} & $\mathrm{Pi}-\mathrm{HCl}$ & $\mathrm{Y}=0,5787+0,0009^{*} * \mathrm{X}$ & 0,99 & 0,88 \\
\hline & P-residual & $\mathrm{Y}=257,30$ & - & 257,30 \\
\hline & $\mathrm{P}$ não-lábil total & $\mathrm{Y}=258,18$ & - & 258,18 \\
\hline & P total & $Y=399,598+0,6054 * * X$ & 0,99 & 601,19 \\
\hline & & \multicolumn{3}{|c|}{ Com calagem } \\
\hline & $\mathrm{Pi}-\mathrm{HCl}$ & $\mathrm{Y}=0,8940+0,0019 * * \mathrm{X}$ & 0,99 & 1,46 \\
\hline & P-residual & $\mathrm{Y}=256,15$ & - & 256,15 \\
\hline & $\mathrm{P}$ não-lábil total & $\mathrm{Y}=257,61$ & - & 257,61 \\
\hline & P total & $\mathrm{Y}=465,721+0,8065 * * \mathrm{X}$ & 0,99 & 706,58 \\
\hline \multirow[t]{10}{*}{ A } & & \multicolumn{3}{|c|}{ Sem calagem } \\
\hline & $\mathrm{Pi}-\mathrm{HCl}$ & $\mathrm{Y}=0,9258+0,0015^{* *} \mathrm{X}$ & 0,99 & 1,41 \\
\hline & P-residual & $\mathrm{Y}=267,21$ & - & 267,21 \\
\hline & P não-lábil total & $Y=268,62$ & - & 268,62 \\
\hline & P total & $\mathrm{Y}=422,25+0,6582 * * \mathrm{X}$ & 0,99 & 649,32 \\
\hline & & \multicolumn{3}{|c|}{ Com calagem } \\
\hline & $\mathrm{Pi}-\mathrm{HCl}$ & $\mathrm{Y}=1,4236+0,0023^{*} * \mathrm{X}$ & 0,99 & 2,22 \\
\hline & P-residual & $\mathrm{Y}=259,66$ & - & 259,66 \\
\hline & $\mathrm{P}$ não-lábil total & $\mathrm{Y}=261,88$ & - & 261,88 \\
\hline & P total & $\mathrm{Y}=403,595+0,5222 * * \mathrm{X}$ & 0,99 & 583,55 \\
\hline \multirow[t]{10}{*}{ GP } & & \multicolumn{3}{|c|}{ Sem calagem } \\
\hline & $\mathrm{Pi}-\mathrm{HCl}$ & $\mathrm{Y}=0,2305+0,00004 * * \mathrm{X}$ & 0,93 & 0,25 \\
\hline & P-residual & $\mathrm{Y}=37,56$ & - & 37,56 \\
\hline & P não-lábil total & $\mathrm{Y}=37,81$ & - & 37,81 \\
\hline & $\mathrm{P}$ total & $\mathrm{Y}=403,595+0,5222$ & 0,99 & 329,11 \\
\hline & & \multicolumn{3}{|c|}{ Com calagem } \\
\hline & $\mathrm{Pi}-\mathrm{HCl}$ & $\mathrm{Y}=0,3778+0,00006^{* *} \mathrm{X}$ & 0,98 & 0,40 \\
\hline & P-residual & $\mathrm{Y}=38,36$ & - & 38,36 \\
\hline & $\mathrm{P}$ não-lábil total & $\mathrm{Y}=38,76$ & - & 38,76 \\
\hline & P total & $\mathrm{Y}=58,0475+0,4993 * * \mathrm{X}$ & 0,99 & 237,30 \\
\hline
\end{tabular}

${ }^{(1)} \mathrm{GH}$ : Glei Húmico; O: Orgânico; A: Aluvial; GP: Glei Pouco Húmico. **Significativo a 1\% de probabilidade pelo teste t. 
solos estudados, independentemente da calagem, variaram de $40,8 \%$ a $55,7 \%$ do $P$ total (soma de todas as frações na dose correspondente a $90 \%$ da produção máxima) (Tabela 6). Quanto ao P não-lábil total (soma das frações de $\mathrm{P}$ pouco lábil na dose correspondente a $90 \%$ da produção máxima), os valores correspondentes à dose de $\mathrm{P}$ com $90 \%$ da produção máxima, tanto na ausência quanto na presença de calagem, variaram de $36,5 \%$ a $51,5 \%$ do P total nos solos GH, O e A, enquanto no solo GP esse valor correspondeu a $11,5 \%$ e $16,3 \%$ do P total, na ausência e presença de calagem, respectivamente (Tabela 6).

$\mathrm{Na}$ presença de calagem, os valores de $\mathrm{P}$ total correlacionaram-se significativa e negativamente com a MSGR, MSTO e P-MSTO, correspondentes à dose de $\mathrm{P}$ com $90 \%$ da produção máxima (Tabela 4); isto indica que essa forma de P não é uma boa característica para prever a disponibilidade de $\mathrm{P}$ para as plantas.

\section{Conclusões}

1. A calagem e a adubação fosfatada influenciam as formas de $\mathrm{P}$ de maneira distinta nos solos de várzea estudados.

2. Exceto no solo Glei Pouco Húmico, as formas de P lábil, tanto na ausência quanto na presença de calagem, têm pouca participação no $P$ total dos solos.

3. Com a neutralização do Al trocável pela calagem, o $\mathrm{P}$ lábil é a forma de $\mathrm{P}$ preferencialmente absorvida pelas plantas de feijoeiro.

\section{Referências}

ALVAREZ, V. H.; FONSECA, D. B. da. Definição de doses de fósforo para determinação da capacidade máxima de adsorção de fosfatos e para ensaios em casa de vegetação. Revista Brasileira de Ciência do Solo, Campinas, v. 14, p. 49-55, 1990.

AMER, G.; BOULDIN, D. R.; BLACK, C. A.; DUKE, F. R. Characterization of soil phosphorus by anion exchange resin adsorption and ${ }^{32} \mathrm{P}$ equilibration. Plant and Soil, Dordrecht, v. 6, p. 391-408, 1955.

ANDRADE, C. A. de B. Limitações de fertilidade e efeito do calcário para o feijoeiro em solos de várzea do sul de Minas Gerais. Lavras: Ufla, 1997. 107 p. Tese de Doutorado.

Pesq. agropec. bras., Brasília, v. 37, n. 3, p. 373-383, mar. 2002
ARAÚJO, M. S. B.; SALCEDO, L. H.; SAMPAIO, V. S. B. Efeito de fertilizações fosfatadas anuais em solo cultivado com cana-de-açúcar. I. intensidade e formas de acumulação. Revista Brasileira de Ciência do Solo, Campinas, v. 17, n. 3, p. 389-396, set./out. 1993.

BECK, M. A. Inorganic and organic phosphorus transformations during 18 years of cultivation in the Amazon basin. Raleigh: North Caroline State University, 1991. 145 p. M.Sc. Dissertation.

BROWMAN, R. A.; COLE, C. V. Transformations of organic phosphorus substrates in soil are evaluated by $\mathrm{NaHCO}_{3}$ extraction. Soil Science, Baltimore, v. 125, n. 1, p. 49-54, Jan. 1978.

CHANG, S. C.; JACKSON, M. L. Fractionation of soil phosphorus. Soil Science, Baltimore, v. 84, p. 133-144, 1957.

EMBRAPA. Centro Nacional de Pesquisa de Solos (Rio de Janeiro, RJ). Manual de métodos de análise de solo. Rio de Janeiro, 1997. 212 p.

FARMER, V. C.; MITCHELL, B. D. Occurrence of oxalates in soil clays following hydrogen peroxide treatment. Soil Science, Baltimore, v. 96, n. 3, p. 221229, Mar. 1963.

FERNANDES, L. A.; FURTINI NETO, A. E.; CURI, N.; LIMA, J. M.; GUEDES, G. A. A. Fósforo e atividade de fosfatase em dois solos sob diferentes condições de uso. Pesquisa Agropecuária Brasileira, Brasília, v. 33, n. 7, p. 1159-1170, jul. 1998.

HEDLEY, M. J.; STEWARD, W. B.; CHAUHAN, B. S. Changes in inorganic and organic soil phosphorus fraction induced by cultivation practices and laboratory incubation. Soil Science Society of America Journal, Madison, v. 46, n. 4, p. 970-976, July/Aug. 1982.

IVANOFF, D. B.; REDDY, K. R.; ROBINSON, S. Chemical fractionation of organic phosphorus in selected histosols. Soil Science, Baltimore, v. 163, n. 1, p. 36-45, Jan. 1998.

LINDSAY, W. L.; MORENO, E. C. Phosphate phase equilibria in soils. Soil Science Society of America Proceedings, Madison, v. 24, n. 1, p. 177-182, Jan./Feb. 1960.

MALAVOLTA, E.; VITTI, G. C.; OLIVEIRA, S. A. Avaliação do estado nutricional das plantas: princípios e aplicações. Piracicaba: Potafos, 1997. 289 p.

MEHRA, O. P.; JACKSON, N. L. Iron oxide removal from soils and clays by a dithionite-citrate system buffered with 
sodium bicarbonate. Clays and Clay Minerals, Boulder, v. 3, p. $317-327,1960$.

MURPHY, J.; RILEY, J. P. A modified single solution method for the determination of phosphate in natural waters. Analytica Chimica Acta, Amsterdam, v. 27, p. 3136, 1962.

NAHAS, E.; CENTURION, J. F.; ASSIS, L. C. Efeito das características dos solos sob os microrganismos solubilizadores de fosfatos e produtores de fosfatases. Revista Brasileira de Ciência do Solo, Campinas, v. 18, n. 1, p. 43-48, jan./abr. 1994.

PAVAN, M. A.; CHAVES, J. C. D. Alterações nas frações de fósforo no solo associadas com a densidade populacional de cafeeiros. Revista Brasileira de Ciência do Solo, Viçosa, v. 20, n. 2, p. 251-256, maio/ago. 1996.

PERROTT, K. W.; MANSELL, G. P. Effect of fertilizer phosphorus and liming on inorganic and organic soil phosphorus fractions. New Zealand Journal of Agricultural Research, Wellington, v. 32, n. 1, p. 63-70, Feb. 1989.

SAH, R. N.; MIKKELSEN, D. S. Effects of temperature and prior flooding on intensity and sorption of phosphate in soil. Plant and Soil, Dordrecht, v. 95, n. 2, p. 163-171, 1986a.

SAH, R. N.; MIKKELSEN, D. S. Sorption and bioavailability of phosphorus during the drainage period of flooded-drained soils. Plant and Soil, Dordrecht, v. 92, n. 2 , p. $265-278,1986 b$.

SAYAL, S.; DE DATTA, S. Chemistry of phosphorus transformations in soil. Advances in Soil Sciences, New York, v. 16, n. 5, p. 2-120, 1991.
SIBBESEN, G. E. A simple ion-exchange resin procedure for extracting plant- available elements from soil. Plant and Soil, Dordrecht, v. 46, n. 3, p. 665-669, Apr. 1977.

SMECK, N. E. Phosphorus dynamics in soils and landscapes. Geoderma, Amsterdam, v. 36, n. 2, p. 185199, Nov. 1985.

STEWART, J. W. B.; TIESSEN, H. Dynamics of soil organic phosphorus. Biogeochemistry, Dordrecht, v. 4, p. 41-60, 1987.

SWENSON, R. M.; COLE, C. V.; SIELING, D. H. Fixation of phosphorus by $\mathrm{Fe}$ and $\mathrm{Al}$ and replacement by organic and inorganic ions. Soil Science, Baltimore, v. 67, n. 1, p. 3-32, Jan./June 1949.

TIESSEN, H.; SALCEDO, I. H.; SAMPAIO, E. V. S. B. Nutrients and soil organic matter dynamics under shifting cultivation in semi-arid northeastern Brazil. Agriculture, Ecosystems and Environments, Amsterdam, v. 38, p. 139-159, 1992.

WALKER, T. W.; SYERS, J. K. The fate of phosphorus during pedogenesis. Geoderma, Amsterdam, v. 15, n. 1, p. 1-19, Jan. 1976.

WANG, C.; FANG, I.; ROBERTSON, L. S. Forms of phosphorus in soils and their availability to sugarcane. In: CONGRESS OF THE INTERNATIONAL SOCIETY OF SUGARCANE TECHNOLOGISTS, 16., 1977, São Paulo. Proceedings... São Paulo: Esalq, 1977. p. 1489-1507.

WILLETT, I. R. The effects of flooding for rice culture on soil chemical properties and subsequent maize growth. Plant and Soil, Dordrecht, v. 52, n. 3, p. 373-383, Aug. 1979.

WILLIANS, J. D. H.; MAYER, T.; NRIAGU, J. O. Extractability of phosphate minerals common in soils and sediments. Soil Science Society of America Journal, Madison, v. 44, n. 3, p. 462-465, May/June 1980. 